\title{
THE CLASSES OF THE QUASIHOMOGENEOUS HILBERT SCHEMES OF POINTS ON THE PLANE
}

\author{
A. BURYAK
}

\begin{abstract}
In this paper we give a formula for the classes (in the Grothendieck ring of complex quasi-projective varieties) of irreducible components of $(1, k)$-quasi-homogeneous Hilbert schemes of points on the plane. We find a new simple geometric interpretation of the $q, t$-Catalan numbers. Finally, we investigate a connection between $(1, k)$-quasi-homogeneous Hilbert schemes and homogeneous nested Hilbert schemes.
\end{abstract}

\section{INTRODUCTION}

The Hilbert scheme $\left(\mathbb{C}^{2}\right)^{[n]}$ of $n$ points in the plane $\mathbb{C}^{2}$ parametrizes the ideals $I \subset \mathbb{C}[x, y]$ of colength $n$ : $\operatorname{dim}_{\mathbb{C}} \mathbb{C}[x, y] / I=n$. There is an open dense subset of $\left(\mathbb{C}^{2}\right)^{[n]}$ that parametrizes the ideals associated with configurations of $n$ distinct points. The Hilbert scheme of $n$ points in the plane is a nonsingular, irreducible, quasiprojective algebraic variety of dimension $2 n$ with a rich and much studied geometry, see [9, 18] for an introduction.

The cohomology groups of $\left(\mathbb{C}^{2}\right)^{[n]}$ were computed in [6] and we refer the reader to the papers [5, 14, 15, 16, 19] for the description of the ring structure in the cohomology $H^{*}\left(\left(\mathbb{C}^{2}\right)^{[n]}\right)$. Let $\bar{n}=\left(n_{1}, \ldots, n_{k}\right)$. The nested Hilbert scheme $\left(\mathbb{C}^{2}\right)^{[\bar{n}]}$ parametrizes $k$-tuples $\left(I_{1}, I_{2}, \ldots, I_{k}\right)$ of ideals $I_{j} \subset \mathbb{C}[x, y]$ such that $I_{j} \subset I_{h}$ for $j<h$ and $\operatorname{dim}_{\mathbb{C}} \mathbb{C}[x, y] / I_{j}=n_{j}$. In [4] J. Cheah studied smoothness and the homology groups of the nested Hilbert schemes $\left(\mathbb{C}^{2}\right)^{[\bar{n}]}$.

There is a $\left(\mathbb{C}^{*}\right)^{2}$-action on $\left(\mathbb{C}^{2}\right)^{[n]}$ that plays a central role in this subject. The algebraic torus $T=\left(\mathbb{C}^{*}\right)^{2}$ acts on $\mathbb{C}^{2}$ by scaling the coordinates, $\left(t_{1}, t_{2}\right)(x, y)=\left(t_{1} x, t_{2} y\right)$. This action lifts to the $T$-action on the Hilbert scheme $\left(\mathbb{C}^{2}\right)^{[n]}$.

Let $T_{a, b}=\left\{\left(t^{a}, t^{b}\right) \in T \mid t \in \mathbb{C}^{*}\right\}$, where $a, b \geq 1$ and $\operatorname{gcd}(a, b)=1$, be a one dimensional subtorus of $T$. Let $\left(\mathbb{C}^{2}\right)_{a, b}^{[n]}$ be the set of fixed points of the $T_{a, b}$-action on the Hilbert scheme $\left(\mathbb{C}^{2}\right)^{[n]}$. The variety $\left(\mathbb{C}^{2}\right)_{a, b}^{[n]}$ is smooth and parameterizes quasi-homogeneous ideals of colength $n$

2010 Mathematics Subject Classification. 14C05, 05A17.

Key words and phrases. Hilbert scheme, torus action, $q$, $t$-Catalan numbers.

The author is partially supported by the grants RFBR-10-01-00678, NSh8462.2010.1, the Vidi grant of NWO and by the Moebius Contest Foundation for Young Scientists. 
in the ring $\mathbb{C}[x, y]$. Irreducible components of $\left(\mathbb{C}^{2}\right)_{1,1}^{[n]}$ were described in [11] and a description of the irreducible components of $\left(\mathbb{C}^{2}\right)_{a, b}^{[n]}$ for arbitrary $a$ and $b$ was obtained in [7].

We denote by $K_{0}\left(\nu_{\mathbb{C}}\right)$ the Grothendieck ring of complex quasiprojective varieties. The classes of the irreducible components of the Hilbert scheme $\left(\mathbb{C}^{2}\right)_{1,1}^{[n]}$ in $K_{0}\left(\nu_{\mathbb{C}}\right)$ were computed in [12].

Let $\left(\mathbb{C}^{2}\right)_{a, b}^{[\bar{n}]}$ be the set of fixed points of the $T_{a, b}$-action on the nested Hilbert scheme $\left(\mathbb{C}^{2}\right)^{[\bar{n}]}$. The dimensions of the irreducible components of $\left(\mathbb{C}^{2}\right)_{1,1}^{[(n, n+1)]}$ were computed in [4].

In this paper we generalize the result of [12] and give a formula for the classes in $K_{0}\left(\nu_{\mathbb{C}}\right)$ of the irreducible components of the variety $\left(\mathbb{C}^{2}\right)_{1, k}^{[n]}$ for an arbitrary positive $k$. As an application, we find an interesting combinatorial identity. We formulate a conjectural formula for the generating series of the classes $\left[\left(\mathbb{C}^{2}\right)_{a, b}^{[n]}\right]$. The combinatorics related to the action of the torus $T_{1, k}$ is very similar to the combinatorics of the $k$-parameter $q, t$-Catalan numbers and we find a new simple geometric interpretation of these numbers.

We also investigate a connection between $(1, k)$-quasi-homogeneous Hilbert schemes and homogeneous nested Hilbert schemes. We construct a natural map $\pi:\left(\mathbb{C}^{2}\right)_{1, k}^{[n]} \rightarrow\left(\mathbb{C}^{2}\right)_{1,1}^{[\bar{n}]}$. We find a sufficient condition for the restriction of this map to an irreducible component to be an isomorphism. In particular, this condition is satisfied when $\bar{n}=(n+1, n)$. Hence, we generalize the result from [4], where the dimensions of the irreducible components in this case were computed.

1.1. Grothendieck ring of quasi-projective varieties. Here we recall a definition of the Grothendieck ring $K_{0}\left(\nu_{\mathbb{C}}\right)$ of complex quasiprojective varieties. It is the abelian group generated by the classes $[X]$ of all complex quasi-projective varieties $X$ modulo the relations:

(1) if varieties $X$ and $Y$ are isomorphic, then $[X]=[Y]$;

(2) if $Y$ is a Zariski closed subvariety of $X$, then $[X]=[Y]+[X \backslash Y]$.

The multiplication in $K_{0}\left(\nu_{\mathbb{C}}\right)$ is defined by the Cartesian product of varieties: $\left[X_{1}\right] \cdot\left[X_{2}\right]=\left[X_{1} \times X_{2}\right]$. The class $\left[\mathbb{A}_{\mathbb{C}}^{1}\right] \in K_{0}\left(\nu_{\mathbb{C}}\right)$ of the complex affine line is denoted by $\mathbb{L}$.

1.2. Description of the irreducible components of $\left(\mathbb{C}^{2}\right)_{a, b}^{[n]}$. Let us recall a description of the irreducible components of the variety $\left(\mathbb{C}^{2}\right)_{a, b}^{[n]}$. Let $\mathbb{C}[x, y]_{a, b}^{d} \subset \mathbb{C}[x, y]$ be the subspace of quasihomogeneous polynomials of degree $d$ with respect to the action of $T_{a, b}$. Let $H=\left(d_{0}, d_{1}, \ldots\right)$ be a sequence of non-negative integers such that $\sum_{i \geq 0} d_{i}=n$. Let $\left(\mathbb{C}^{2}\right)_{a, b}^{[n]}(H) \subset\left(\mathbb{C}^{2}\right)_{a, b}^{[n]}$ be the set of points corresponding to quasihomogeneous ideals $I \subset \mathbb{C}[x, y]$ such that $\operatorname{dim}\left(\mathbb{C}[x, y]_{a, b}^{i} /\left(I \cap \mathbb{C}[x, y]_{a, b}^{i}\right)\right)=d_{i}$. 
Proposition $1.1([7])$. If $\left(\mathbb{C}^{2}\right)_{a, b}^{[n]}(H) \neq \emptyset$, then $\left(\mathbb{C}^{2}\right)_{a, b}^{[n]}(H)$ is an irreducible component of $\left(\mathbb{C}^{2}\right)_{a, b}^{[n]}$.

1.3. Classes of the irreducible components of $\left(\mathbb{C}^{2}\right)_{1, k}^{[n]}$. In this section we fix $k \geq 1$. For numbers $M, N \geq 0$ let $G(M, N)_{q}=\frac{\prod_{i=1}^{M+N}\left(1-q^{i}\right)}{\prod_{i=1}^{M}\left(1-q^{i}\right) \prod_{i=1}^{N}\left(1-q^{i}\right)}$. Let $\eta(H)$ be the largest $i$, such that $d_{i}=\left[\frac{i}{k}\right]+1$. We adopt the following conventions, $\eta(H)=-1$, if $H=(0,0, \ldots) ; d_{-1}=0$. We introduce an auxiliary function $\tau$ defined by the following rule, $\tau(i)=1$, if $k \mid i+1$ and $\tau(i)=0$, if $k \nmid i+1$. We will prove the following statement.

Theorem 1.2. Let $H=\left(d_{0}, d_{1}, \ldots\right), n=\sum_{i \geq 0} d_{i}$. If $\left(\mathbb{C}^{2}\right)_{1, k}^{[n]}(H) \neq \emptyset$, then

$$
\left[\left(\mathbb{C}^{2}\right)_{1, k}^{[n]}(H)\right]=\prod_{i \geq \eta} G\left(d_{i}-d_{i+1}+\tau(i), d_{i+1}-d_{i+1+k}\right)_{\mathbb{L}} .
$$

Remark 1.3. We see that the classes of the irreducible components of $\left(\mathbb{C}^{2}\right)_{1, k}^{[n]}$ are polynomials in $\mathbb{L}$. Moreover, all roots of these polynomials are the roots of unity. In the case of an arbitrary pair $(a, b)$, this is not true. For example, it is easy to compute that

$$
\left[\left(\mathbb{C}^{2}\right)_{2,3}^{[12]}(1,0,1,1,1,1,2,1,1,1,1,0,1)\right]=1+3 \mathbb{L}+\mathbb{L}^{2} .
$$

1.4. Conjecture. The following conjectural formula for the generating series of the classes $\left[\left(\mathbb{C}^{2}\right)_{a, b}^{[n]}\right]$ is based on computer calculations.

\section{Conjecture 1.4.}

$$
\sum_{n \geq 0}\left[\left(\mathbb{C}^{2}\right)_{a, b}^{[n]}\right] t^{n}=\prod_{\substack{i \geq 1 \\(a+b) \nmid i}} \frac{1}{1-t^{i}} \prod_{i \geq 1} \frac{1}{1-\mathbb{L} t^{(a+b) i}} .
$$

Similar conjectural formulas for the generating series of the classes of some equivariant Hilbert schemes can be found in [8].

1.5. Definition of the $(q, t)$-Catalan numbers. A $k$-Dyck path is a lattice path from $(0,0)$ to $(k n, n)$ consisting of $(0,1)$ and $(1,0)$ steps, never going below the line $x=k y$ (see Figure 2). Let $L_{k n, n}^{+}$denote the set of these paths. For a $k$-path $\pi$ let $D_{\pi}^{\prime}$ be the set of squares which are above $\pi$ and contained in the rectangle with vertices $(0,0),(k n, 0)$, $(k n, n)$ and $(0, n)$. The set $D_{\pi}^{\prime}$ reflected with respect to the horizontal line is a Young diagram. We denote it by $D_{\pi}$.

For a Young diagram $D$ and a box $s \in D$ let $a(s)$ denote the number of boxes in $D$ in the same column and strictly above $s$ and let $l(s)$ denote the number of boxes in $D$ in the same row and strictly right of $s$ (see Figure 1). 


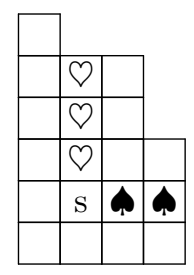

$$
\begin{aligned}
& l(s)=\text { number of } \boldsymbol{\phi} \\
& a(s)=\text { number of } \varnothing
\end{aligned}
$$

\section{FiguRE 1.}

For a $k$-path $\pi$ let area $(\pi)$ be the number of full squares below $\pi$ and above the line $k y=x$, and let

$$
b_{k}(\pi)=\left|\left\{s \in D_{\pi} \mid k a(s) \leq l(s) \leq k(a(s)+1)\right\}\right| .
$$

An example is on Figure 2,
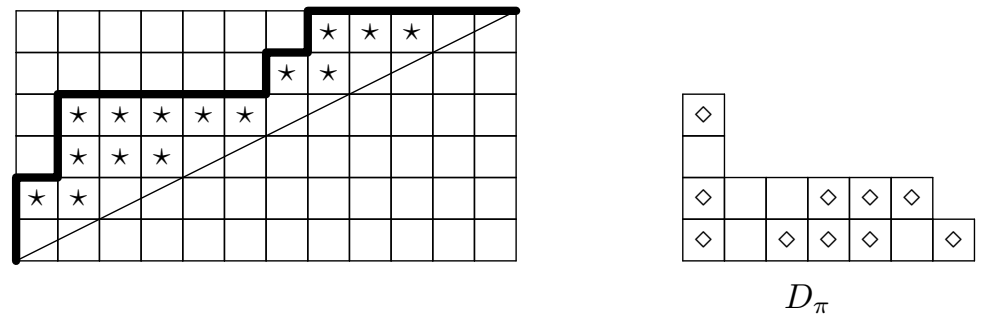

FiguRE 2. A 2-path $\pi$ with $\operatorname{area}(\pi)=15$ and $b_{k}(\pi)=$ 10 (contributors to $b_{k}(\pi)$ are marked by $\diamond$, and those to $\operatorname{area}(\pi)$ by $\star)$.

The combinatorial $k$-parameter $(q, t)$-Catalan number is defined by the formula

$$
C_{n}^{(k)}(q, t)=\sum_{\pi \in L_{k n, n}^{+}} q^{b_{k}(\pi)} t^{\text {area }(\pi)} .
$$

We refer the reader to the book [10] for another equivalent beautiful definitions of the $q, t$-Catalan numbers.

1.6. $(q, t)$-Catalan numbers and the Hilbert schemes. Let $V_{k, n}$ be the vector subspace of $\mathbb{C}[x, y]$ generated by the monomials $x^{i} y^{j}$ with $i+k j \leq k n-k-1$. Let $\left(\mathbb{C}^{2}\right)^{[N](k, n)}$ be the subset of $\left(\mathbb{C}^{2}\right)^{[N]}$ that parametrizes ideals $I \subset \mathbb{C}[x, y]$ such that $I+V_{k, n}=\mathbb{C}[x, y]$. It is easy to see that $\left(\mathbb{C}^{2}\right)^{[N](k, n)}$ is an open subset of the variety $\left(\mathbb{C}^{2}\right)^{[N]}$.

\section{Theorem 1.5.}

$$
\sum_{N \geq 0}\left[\left(\mathbb{C}^{2}\right)^{[N](k, n)}\right] t^{N}=(\mathbb{L} t)^{\frac{k n(n-1)}{2}} C_{n}^{(k)}\left(\mathbb{L}, \mathbb{L}^{-1} t^{-1}\right) .
$$


1.7. Combinatorial identity. We say that a sequence $H=\left(d_{0}, d_{1}, \ldots\right)$ is good if for any $i \geq \eta(H)$ we have $d_{i}-d_{i+1}+\tau(i) \geq 0$ and $d_{i+1} \leq$ $d_{i+1-k}$.

\section{Theorem 1.6.}

$$
\sum_{\{\text {good } H\}} \prod_{i \geq \eta} G\left(d_{i}-d_{i+1}+\tau(i), d_{i+1}-d_{i+1+k}\right)_{q} q^{\chi(H)} t^{\sum d_{i}}=\prod_{i \geq 1} \frac{1}{1-q t^{i}}
$$

where

$$
\begin{aligned}
& \chi(H)=\sum_{i \geq \eta}\left(d_{i}-d_{i+1}+\tau(i)\right) \times \\
& \times\left(\frac{k}{2}\left(d_{i}-d_{i+1}+\tau(i)-1\right)+\sum_{j=1}^{k-1}(k-j)\left(d_{i+j}-d_{i+j+1}+\tau(i+j)\right)\right) .
\end{aligned}
$$

In the case $k=1$ this identity was proved in [13].

1.8. Homogeneous nested Hilbert schemes. Let $\bar{n}=\left(n_{1}, n_{2}, \ldots, n_{k}\right)$, where $n_{1}, \ldots, n_{k}$ are non-negative integers such that $n_{1} \geq n_{2} \geq \ldots \geq$ $n_{k}$. Let $\bar{H}=\left(H_{1}, H_{2}, \ldots, H_{k}\right)$, where $H_{i}=\left(d_{i, 0}, d_{i, 1}, \ldots\right)$ and $\sum_{j \geq 0} d_{i, j}=$ $n_{i}$. Let $\left(\mathbb{C}^{2}\right)_{a, b}^{[\bar{n}]}(\bar{H})=\left\{\left(Z_{1}, \ldots, Z_{k}\right) \in\left(\mathbb{C}^{2}\right)^{[\bar{n}]} \mid Z_{i} \in\left(\mathbb{C}^{2}\right)_{a, b}^{\left[n_{i}\right]}\left(H_{i}\right)\right\}$. Let $E(\bar{H})=\left\{i \in \mathbb{Z}_{\geq 0} \mid d_{1, i}=d_{2, i}=\ldots=d_{k, i}\right\}, n=\sum_{i=1}^{k} n_{i}$ and $H=$ $\left(d_{0}, d_{1}, \ldots\right)$, where $d_{i+k j}=d_{i+1, j}, 0 \leq i<k, j \geq 0$. We will prove the following statement.

Theorem 1.7. Suppose that for any two numbers $i, j \in \mathbb{Z}_{>0} \backslash E(\bar{H}), i<$ $j$, we have $j-i \geq 2$. Then the variety $\left(\mathbb{C}^{2}\right)_{1,1}^{[\bar{n}]}(\bar{H})$ is isomorphic to $\left(\mathbb{C}^{2}\right)_{1, k}^{[n]}(H)$.

1.9. Organization of the paper. In section 2 we construct a cellular decomposition of the quasihomogeneous Hilbert scheme and reduce Theorem 1.2 to a combinatorial identity. In section 3 we construct a bijection that is a generalization of the hook code from [12]. The main result of this section is Proposition 3.7. Finally, in section 4 we apply it to conclude the proof of Theorem [1.2. The proof of Theorem [1.5] is in section 5. We prove Theorem 1.6 in section 6. Section 7 contains the proof of Theorem 1.7 .

1.10. Acknowledgments. The author is grateful to S. M. GuseinZade for suggesting the area of research. The author is grateful to B. L. Feigin and A. N. Kirillov who noticed that the main result from [12] can be generalized to the $(1, k)$-case and suggested the author to work on this problem. The author is grateful to S. Shadrin, M. Kazarian, S. Lando and A. Oblomkov for useful discussions. 


\section{Cellular Decomposition of $\left(\mathbb{C}^{2}\right)_{1, k}^{[n]}$}

In this section we reduce Theorem 1.2 to the combinatorial identity (4) using a cellular decomposition of $\left(\mathbb{C}^{2}\right)_{1, k}^{[n]}$.

Consider the $T$-action on $\left(\mathbb{C}^{2}\right)^{[n]}$. Fixed points of this action correspond to monomial ideals in $\mathbb{C}[x, y]$. Let $I \subset \mathbb{C}[x, y]$ be a monomial ideal of colength $n$. Let $D_{I}=\left\{(i, j) \in \mathbb{Z}_{>0}^{2} \mid x^{i} y^{j} \notin I\right\}$ be the corresponding Young diagram. We will use the following notations. For a Young diagram $D$ let

$$
\begin{aligned}
& r_{l}(D)=|\{(i, j) \in D \mid j=l\}|, \\
& c_{l}(D)=|\{(i, j) \in D \mid i=l\}|, \\
& \operatorname{diag}_{l}^{a, b}(D)=|\{(i, j) \in D \mid a i+b j=l\}|, \\
& \operatorname{diag}^{a, b}(D)=\left(\operatorname{diag}_{0}^{a, b}(D), \operatorname{diag}_{1}^{a, b}(D), \operatorname{diag}_{2}^{a, b}(D), \ldots\right) .
\end{aligned}
$$

Let $p \in\left(\mathbb{C}^{2}\right)^{[n]}$ be the fixed point corresponding to a Young diagram $D$. Let $R(T)=\mathbb{Z}\left[t_{1}, t_{2}\right]$ be the representation ring of $T$. Then the weight decomposition of $T_{p}\left(\mathbb{C}^{2}\right)^{[n]}$ is given by (see [6])

$$
T_{p}\left(\mathbb{C}^{2}\right)^{[n]}=\sum_{s \in D}\left(t_{1}^{l(s)+1} t_{2}^{-a(s)}+t_{1}^{-l(s)} t_{2}^{a(s)+1}\right) .
$$

Obviously, the variety $\left(\mathbb{C}^{2}\right)_{1, k}^{[n]}$ is invariant under the $T$-action and contains all fixed points of the $T$-action on $\left(\mathbb{C}^{2}\right)^{[n]}$. Hence, the weight decomposition of $T_{p}\left(\mathbb{C}^{2}\right)_{1, k}^{[n]}$ is given by

$$
T_{p}\left(\mathbb{C}^{2}\right)_{1, k}^{[n]}=\sum_{\substack{s \in D \\ l(s)+1=k a(s)}} t_{1}^{l(s)+1} t_{2}^{-a(s)}+\sum_{\substack{s \in D \\ l(s)=k(a(s)+1)}} t_{1}^{-l(s)} t_{2}^{a(s)+1} .
$$

Consider the $T_{1, \alpha}$-action on $\left(\mathbb{C}^{2}\right)_{1, k}^{[n]}$, where $\alpha$ is a positive integer. If $\alpha$ is big enough then the set of fixed points of the $T_{1, \alpha}$-action coincides with the set of fixed points of the $T$-action. For a fixed point $p \in\left(\mathbb{C}^{2}\right)_{1, k}^{[n]}$ let $C_{p}=\left\{z \in\left(\mathbb{C}^{2}\right)_{1, k}^{[n]} \mid \lim _{t \rightarrow 0, t \in T_{1, \alpha}} t z=p\right\}$. The variety $\left(\mathbb{C}^{2}\right)_{1, k}^{[n]}$ has a cellular decomposition with the cells $C_{p}$ (see [2, 3]). Therefore, the cells $C_{p}$ are isomorphic to affine spaces. It is easy to compute that if a point $p$ corresponds to a Young diagram $D$, then $\operatorname{dim}\left(C_{p}\right)=\mid\{s \in D \mid l(s)=$ $k(a(s)+1)\} \mid$. Moreover, $p \in\left(\mathbb{C}^{2}\right)_{1, k}^{[n]}(H) \Leftrightarrow \operatorname{diag}^{1, k}(D)=H$, where $H=\left(d_{0}, d_{1}, \ldots\right)$ is an arbitrary sequence of non-negative integers.

Let $\mathcal{D}$ be the set of Young diagrams. We see that

$$
\left[\left(\mathbb{C}^{2}\right)_{1, k}^{[n]}(H)\right]=\sum_{\substack{D \in \mathcal{D} \\ \operatorname{diag}^{1, k}(D)=H}} \mathbb{L}^{|\{s \in D \mid l(s)=k(a(s)+1)\}|} .
$$


Therefore, Theorem 1.2 follows from the combinatorial identity:

(3)

$$
\sum_{\substack{D \in \mathcal{D} \\ \operatorname{diag}^{1, k}(D)=H}} q^{|\{s \in D \mid l(s)=k(a(s)+1)\}|}=\prod_{i \geq \eta} G\left(d_{i}-d_{i+1}+\tau(i), d_{i+1}-d_{i+1+k}\right)_{q} .
$$

It is not hard to check that this identity is equivalent to the following identity

$$
\begin{aligned}
& \sum_{\substack{D \in \mathcal{D} \\
\operatorname{diag}^{1, k}(D)=H}} q^{|\{s \in D \mid l(s)=k(a(s)+1)\}|}= \\
& \quad=\frac{1-q}{1-q^{d_{\eta-k+1}+1-d_{\eta+1}}} \prod_{i \geq \eta+1} G\left(d_{i}-d_{i+1}+\tau(i), d_{i-k}-d_{i}\right)_{q} .
\end{aligned}
$$

Here we adopt the following conventions, $d_{i}=0$, if $-k \leq i \leq-1$ and $d_{-k-1}=-1$.

Remark 2.1. Combinatorial constructions from the paper [17] can be used to prove (3). However, our constructions are different from them.

\section{BiJECTION}

In this section we show how to encode an element of the set $\{D \in$ $\left.\mathcal{D} \mid \operatorname{diag}^{1, k}(D)=H\right\}$ as a sequence of partitions. The main result of this section is Proposition 3.7. In section 3.1 we define a map $F$ from the set $\left\{D \in \mathcal{D} \mid \operatorname{diag}^{1, k}(D)=H\right\}$ to the set of sequences $\left(P_{0}, P_{1}, \ldots\right)$, where $P_{i}$ are Young diagrams. In section 3.2 we prove the main properties of the map $F$. In section 3.3 we prove an injectivity of the map $F$ and in section 3.4 we describe the image of $F$.

In this section we fix an arbitrary sequence $H=\left(d_{0}, d_{1}, \ldots\right)$ of nonnegative integers.

3.1. The definition of the map $F$. For a Young diagram $D$ let

$$
\begin{aligned}
& B_{m}(D)=\left\{j \in \mathbb{Z}_{\geq 0} \mid r_{j}(D) \neq 0, k j+r_{j}(D)-1=m\right\}, \\
& h_{m}(D)=|\{s=(i, j) \in D \mid j=m, l(s)=k(a(s)+1)\}| .
\end{aligned}
$$

Let $B_{m}(D)=\left\{j_{1}, j_{2}, \ldots\right\}$, where $j_{1} \leq j_{2} \leq \ldots$ Then $h_{j_{1}}(D) \geq$ $h_{j_{2}}(D) \geq \ldots$, and we denote the partition $\left(h_{j_{1}}(D), h_{j_{2}}(D), \ldots\right)$ by $\lambda(D, m)$.

For a partition $\lambda=\lambda_{0}, \ldots, \lambda_{r}, \lambda_{0} \geq \ldots \geq \lambda_{r}$ let $D_{\lambda}=\{(i, j) \in$ $\left.\mathbb{Z}_{\geq 0}^{2} \mid i \leq r, j \leq \lambda_{i}-1\right\}$ be the corresponding Young diagram. Let $\theta(H)$ be the largest $i \leq \eta(H)$ such that $i \equiv k-1 \bmod k$.

Let $D$ be a Young diagram such that $\operatorname{diag}^{1, k}(D)=H$. We denote by $F(D)$ a sequence of Young diagrams $\left(F(D)_{0}, F(D)_{1}, \ldots\right)$ such that $F(D)_{i}=D_{\lambda(D, i+\theta)}$.

We give an example in Figure 3. We write the number $i+k j$ into the box $(i, j) \in D$ for the reader's convenience. 


$$
\begin{array}{ll}
k=2 & B_{9}(D)=\emptyset \\
H=(1,1,2,2,3,3,4,4,5,5,5,3,2,0,0, \ldots) & B_{10}(D)=\{2,3\} \\
\theta(H)=9 & B_{11}(D)=\{1\} \\
& B_{12}(D)=\{0,4\}
\end{array}
$$

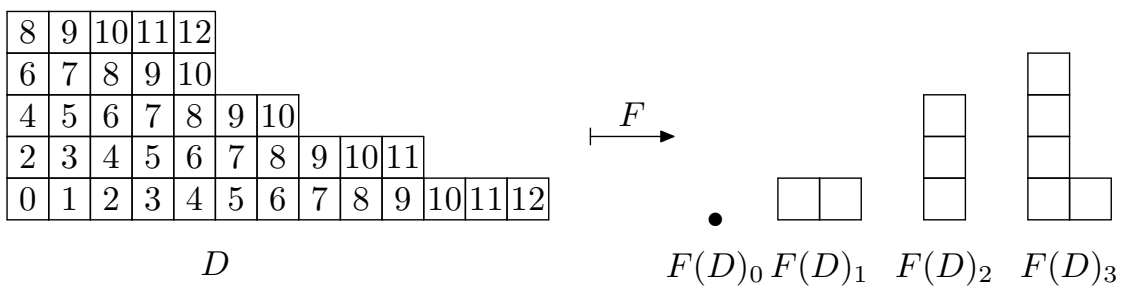

\section{FiguRE 3.}

3.2. The main properties of $F$. We use the following notations:

$$
\begin{aligned}
& w_{i}(H)=d_{i-k+\theta}-d_{i+\theta}+1, \\
& f_{i}(H)= \begin{cases}d_{i+\theta}-d_{i+1+\theta}, & \text { if } k \nmid i, \\
d_{i+\theta}-d_{i+1+\theta}+1, & \text { if } k \mid i .\end{cases}
\end{aligned}
$$

We denote by $R(M, N)$ the rectangle in the integral lattice defined by $R(M, N)=\left\{(i, j) \in \mathbb{Z}_{\geq 0}^{2} \mid i \leq M-1, j \leq N-1\right\}$. We denote by $\mathcal{D}(M, N)$ the set $\{D \in \mathcal{D} \mid D \subset R(M, N)\}$.

Lemma 3.1. The Young diagram $F(D)_{i}$ lies in the rectangle $R\left(f_{i}, w_{i}\right)$.

Proof. Consider a point $(i, j) \in D$. Let $i+k j=l$. Suppose $k \nmid l$, then $(i-1, j) \in D$ and $j \notin B_{l-1}(D)$. Hence, $\left|B_{l-1}(D)\right|=d_{l-1}-d_{l}$. Suppose $k \mid l$. If $i \neq 0$, then $(i-1, j) \in D$ and $j \notin B_{l-1}(D)$. Hence, $\left|B_{l-1}(D)\right| \leq$ $d_{l-1}-d_{l}+1$. Thus, we have proved that $r_{0}\left(F(D)_{l-1-\theta}\right) \leq f_{l-1-\theta}$.

Consider a number $a \in B_{l}(D)$. Let $d_{m}^{\prime}=\mid\{(i, j) \in D \mid j \geq a, i+k j=$ $m\} \mid$. Clearly, $h_{a}=d_{l-k}^{\prime}-d_{l}^{\prime}+1 \leq d_{l-k}-d_{l}+1$. This proves that $c_{0}\left(F(D)_{l-\theta}\right) \leq w_{l-\theta}$.

The following statement describes an important property of the numbers $w_{i}(H)$ and $f_{i}(H)$.

Lemma 3.2. The set $\left\{D \in \mathcal{D} \mid \operatorname{diag}^{1, k}(D)=H\right\}$ is not empty if and only if for any $i>\eta-\theta$ the following condition holds: $f_{i} \geq 0, w_{i} \geq 1$.

Proof. It is easy to check that the set $\left\{D \in \mathcal{D} \mid \operatorname{diag}^{1, k}(D)=H\right\}$ is not empty if and only if for any $i>\eta$ the following three conditions hold: 1) $d_{i} \leq d_{i-k}$;2) if $k \nmid i$, then $d_{i} \leq d_{i-1}$;3) if $k \mid i$, then $d_{i} \leq d_{i-1}+1$. These conditions are equivalent to the condition of the lemma.

Consider a sequence of Young diagrams $P=\left(P_{0}, P_{1}, \ldots\right)$ such that $P_{i} \in \mathcal{D}\left(f_{i}, w_{i}\right)$ (a short notation for that will be $P \in \prod_{i \geq 0} \mathcal{D}\left(f_{i}, w_{i}\right)$ ). Let $\nu(P)$ be the largest $i$ such that $c_{0}\left(P_{i}\right)=w_{i}$. The number $\nu(P)$ is well-defined since $w_{0}=0$, but it can be equal to $\infty$. It is easy to see that if $P=F(D)$, then $\nu(P)<\infty$. 
Lemma 3.3. Let $D$ be a Young diagram such that $\operatorname{diag}^{1, k}(D)=H$. Then $r_{0}(D)=\theta(H)+\nu(F(D))+1$.

Proof. Consider a number $a \in B_{l}(D)$. Suppose that $h_{a}(D)=d_{l-k}-$ $d_{l}+1$. Then for any $0 \leq j \leq a$ we have $\left(r_{a}(D)-1+k j, a-j\right) \in D$. In particular, $(0, l) \in D$. Hence $r_{0}(D) \geq l+1$. On the other hand, $h_{0}(D)=d_{r_{0}(D)-1-k}-d_{r_{0}(D)-1}+1$. This completes the proof of the lemma.

For a Young diagram $D$ let $D(a, b)=\left\{(i, j) \in \mathbb{Z}_{\geq 0}^{2} \mid(i+a, j+b) \in\right.$ $D\}$. Consider an arbitrary Young diagram $D$ such that $\operatorname{diag}^{1, k}(D)=$ $H$. Let $D^{\prime}=D(0,1), H^{\prime}=\operatorname{diag}^{1, k}\left(D^{\prime}\right), F(D)=\left(P_{0}, P_{1}, \ldots\right), F\left(D^{\prime}\right)=$ $\left(P_{0}^{\prime}, P_{1}^{\prime}, \ldots\right), f_{i}^{\prime}=f_{i}\left(H^{\prime}\right), w_{i}^{\prime}=w_{i}\left(H^{\prime}\right), \theta^{\prime}=\theta\left(H^{\prime}\right), \nu=\nu(P), \nu^{\prime}=$ $\nu\left(P^{\prime}\right)$.

Lemma 3.4.

$$
d_{i}^{\prime}= \begin{cases}d_{i+k}-1, & \text { if } i+k \leq \nu+\theta \\ d_{i+k}, & \text { if } i+k>\nu+\theta\end{cases}
$$

1) If $\nu \geq k$ or $w_{k} \geq 2$, then

$$
\begin{aligned}
& \theta^{\prime}=\theta-k ; P_{i}^{\prime}= \begin{cases}P_{i}, & \text { if } i \neq \nu, \\
P_{i}(1,0), & \text { if } i=\nu ;\end{cases} \\
& f_{i}^{\prime}=\left\{\begin{array}{ll}
f_{i}, & \text { if } i \neq \nu, \\
f_{i}-1, & \text { if } i=\nu ;
\end{array} \quad w_{i}^{\prime}= \begin{cases}w_{i}, & \text { if } i \notin[\nu+1, \nu+k], \\
w_{i}-1, & \text { if } i \in[\nu+1, \nu+k] ;\end{cases} \right.
\end{aligned}
$$

2) If $\nu \leq k-1$ and $w_{k}=1$, then

$$
\begin{array}{ll}
\theta^{\prime}=\theta ; & P_{i}^{\prime}=P_{i}+k ; \\
f_{i}^{\prime}=f_{i+k} ; & w_{i}^{\prime}= \begin{cases}w_{i}, & \text { if } i>\nu, \\
w_{i}-1, & \text { if } i \leq \nu .\end{cases}
\end{array}
$$

Proof. The proof is clear from Lemma 3.3 and the definition of the map F.

\subsection{Injectivity of $F$.}

Lemma 3.5. The map $F:\left\{D \in \mathcal{D} \mid \operatorname{diag}^{1, k}(D)=H\right\} \rightarrow \prod_{i \geq 0} \mathcal{D}\left(f_{i}, w_{i}\right)$ is injective.

Proof. The proof is by induction on $|D|$. For $|D|=0$, there is nothing to prove. Assume that $|D|>0$. Using Lemma 3.4, we can reconstruct $F\left(D^{\prime}\right)$. By the inductive assumption, we can reconstruct $D^{\prime}$. From Lemma 3.3 it follows that $F(D)$ determines $r_{0}(D)$. The diagram $D^{\prime}$ and the number $r_{0}(D)$ determines $D$. This completes the proof of the lemma. 
3.4. The image of $F$. Consider a sequence $P \in \prod_{i>0} \mathcal{D}\left(f_{i}, w_{i}\right)$. For a number $i \geq 0$ let $\Phi_{P}(i)$ be the minimal $j>i$ such that $r_{0}\left(P_{j}\right)<f_{j}$. If for any $j>i$ we have $r_{0}\left(P_{j}\right)=f_{j}$, then we put $\Phi_{P}(i)=\infty$.

Lemma 3.6. Let $D$ be a Young diagram such that $\operatorname{diag}^{1, k}(D)=H$, then for any $i \geq 0$ we have $\Phi_{F(D)}(i)-i \leq k$.

Proof. The proof is by induction on $|D|$. For $|D|=0$, there is nothing to prove. Assume that $|D|>0$. We use the notations of Lemma 3.4. Suppose that $\nu>\eta-\theta$ or $\nu=\eta-\theta, f_{\eta-\theta} \geq 2$. From Lemma 3.4 it follows that for any $i \geq 0$ we have $r_{0}\left(P_{i}\right)<f_{i} \Leftrightarrow r_{0}\left(P_{i}^{\prime}\right)<f_{i}^{\prime}$. Thus, Lemma 3.6 follows from the inductive assumption. Assume that $\nu=\eta-\theta$ and $f_{\eta-\theta}=1$. From Lemma 3.4 it follows that we must only prove that $\Phi_{P}(\eta-\theta)-(\eta-\theta) \leq k$. Assume the converse. Clearly, $w_{\eta-\theta+1}=1$. From the definition of the number $\nu$ and the assumption $\Phi_{P}(\eta-\theta)-(\eta-\theta)>k$ it follows that $f_{\eta-\theta+1}=0$. Continuing in the same way, we see that $w_{\eta-\theta+1}=w_{\eta-\theta+2}=\ldots=w_{\eta-\theta+k}=1$ and $f_{\eta-\theta+1}=f_{\eta-\theta+2}=\ldots=f_{\eta-\theta+k}=0$. Clearly, $w_{\eta-\theta+k+1}=0$, but this contradicts Lemma 3.2.

Proposition 3.7. Suppose $\left\{D \in \mathcal{D} \mid \operatorname{diag}^{1, k}(D)=H\right\} \neq \emptyset$, then the map

$$
F:\left\{D \in \mathcal{D} \mid \operatorname{diag}^{1, k}(D)=H\right\} \rightarrow\left\{\begin{array}{l|l}
P \in \prod_{i \geq 0} \mathcal{D}\left(f_{i}, w_{i}\right) & \begin{array}{c}
\forall i \geq 0: \\
\Phi_{P}(i)-i \leq k
\end{array}
\end{array}\right\} .
$$

is a bijection such that $|\{s \in D \mid l(s)=k(a(s)+1)\}|=\sum_{i \geq 0}\left|F(D)_{i}\right|$.

Proof. The second statement of the proposition is clear from the definition of the map $F$. Let us prove that $F$ is a bijection. We have already proved the injectivity. Let us prove the surjectivity of the map $F$. The proof is by induction on $n=\sum_{i>0} d_{i}$. For $n=0$, there is nothing to prove. Assume that $n \geq 1$. Consider a sequence $P \in \prod_{i>0} \mathcal{D}\left(f_{i}, w_{i}\right)$ such that for any $i \geq 0$ we have $\Phi_{P}(i)-i \leq k$. Define $H^{\prime}$ and $P^{\prime}$ by formulas from Lemma 3.4 .

We want to apply the inductive assumption to the sequence $H^{\prime}$, so we need to check that the set $\left\{D \in \mathcal{D} \mid \operatorname{diag}^{1, k}(D)=H^{\prime}\right\}$ is not empty. If $\nu=\eta-\theta$, then it easily follows from Lemma 3.2. Assume that $\nu>\eta-\theta$. By Lemmas 3.4 and [3.2, we must only prove that for any $\nu<i \leq \nu+k$ we have $w_{i} \geq 2$. Assume the converse. Hence, there exists a number $\nu<i \leq \nu+k$ such that $w_{i}=1$. Therefore, $\sum_{j=1}^{k} f_{i-j}=1$. Hence, $\Phi_{P}(i-k-1)=i$. This contradicts the condition $\Phi_{P}(i-k-1)-(i-k-1) \leq k$. Thus, we have prove that $\{D \in$ $\left.\mathcal{D} \mid \operatorname{diag}^{1, k}=H^{\prime}\right\} \neq \emptyset$.

By the inductive assumption, there exists a Young diagram $D^{\prime}$ such that $\operatorname{diag}^{1, k}\left(D^{\prime}\right)=H^{\prime}$ and $F\left(D^{\prime}\right)=P^{\prime}$. Let us prove that $r_{0}\left(D^{\prime}\right) \leq$ $\nu+\theta+1$. By Lemma 3.3, it is equivalent to $\nu^{\prime}+\theta^{\prime} \leq \nu+\theta$ and it follows from Lemma 3.4 . 
Let $D$ be the diagram obtained from $D^{\prime}$ by adding the row of length $\nu+\theta+1$. Clearly, $F(D)=P$.

\section{Proof of Theorem 1.2}

In this section we prove (41) using Proposition 3.7 .

We fix a sequence $H=\left(d_{0}, d_{1}, \ldots\right)$ such that the set $\left\{D \in \mathcal{D} \mid \operatorname{diag}^{1, k}(D)=\right.$ $H\}$ is not empty. We will use the following well known fact (see e.g. [1])

$$
\sum_{D \in \mathcal{D}(M, N)} q^{|D|}=G(M, N)
$$

Let $S(H)=\left\{P \in \prod_{i \geq 0} \mathcal{D}\left(f_{i}, w_{i}\right) \mid \forall i \geq 0: \Phi_{P}(i)-i \leq k\right\}$. Using Proposition 3.7 and our notations we see that (4) is equivalent to the following formula

$$
\sum_{P \in S(H)} q^{|P|}=\frac{1-q}{1-q^{w_{\eta-\theta+1}}} \prod_{i \geq \eta-\theta+1} G\left(f_{i}, w_{i}-1\right),
$$

where $|P|=\sum_{i>0}\left|P_{i}\right|$. Let $\sigma(H)$ be the minimal $i \geq 0$ such that for any $j>\theta+i$ we have $d_{j}=0$. Let $\psi(H)$ be the maximal $i \leq \sigma(H)$ such that $k \mid i$. For a sequence $P \in S(H)$ let $\phi_{P}(i)$ be the maximal $j<i$ such that $r_{0}\left(P_{j}\right)<f_{j}$. We claim that

$$
\sum_{\substack{P \in S(H) \\ \phi_{P}(\psi+k)=p}} q^{|P|}=q^{\sum_{i=p+1}^{\psi+k-1} f_{i}} \frac{1-q^{f_{p}}}{1-q^{w_{\psi+k}}}\left(\sum_{P \in S(H)} q^{|P|}\right),
$$

where $\psi \leq p<\psi+k$.

Let us prove (15) and (6) by induction on $\sigma$. Suppose $\sigma<k$, then

$$
\sum_{P \in S(H)} q^{|P|}=\prod_{i=\eta-\theta+1}^{k-1} G\left(f_{i}, w_{i}\right)=\frac{1-q}{1-q^{w_{\eta-\theta+1}}} \prod_{i \geq \eta-\theta+1} G\left(f_{i}, w_{i}-1\right) .
$$

Hence, (5) is proved. It is clear that

$$
\begin{aligned}
\sum_{\substack{P \in S(H) \\
\phi_{P}(k)=p}} q^{|P|} & =\prod_{i=\eta-\theta+1}^{p} G\left(f_{i}-\delta_{i}^{p}, w_{i}\right) \prod_{i=p+1}^{k-1} q^{f_{i}} G\left(f_{i}, w_{i}-1\right)= \\
& =q^{\sum_{i=p+1}^{k-1} f_{i}} \frac{1-q^{f_{p}}}{1-q^{w_{k}}}\left(\frac{1-q}{1-q^{w_{\eta-\theta+1}}} \prod_{i \geq \eta-\theta+1} G\left(f_{i}, w_{i}-1\right)\right) .
\end{aligned}
$$

Therefore, (6) is proved.

Suppose $\sigma \geq k$. For $p>\eta(H)$ let

$$
\begin{aligned}
& H(p)=\left(d_{0}(p), d_{1}(p), d_{2}(p), \ldots\right), \text { where } \\
& d_{i}(p)= \begin{cases}d_{k d_{p+1}+i}-d_{p+1}, & \text { if } k d_{p+1}+i \leq p, \\
0, & \text { if } k d_{p+1}+i>p .\end{cases}
\end{aligned}
$$


If $d_{p} \geq d_{p+1}$, then $\left\{D \in \mathcal{D} \mid \operatorname{diag}^{1, k}(D)=H(p)\right\} \neq \emptyset$. We adopt the following convention, $S(H(p))=\emptyset$, if $d_{p}<d_{p+1}$. Note that if $d_{p}<d_{p+1}$, then $k \mid p+1$. Let $H^{\prime}=H(\theta+\sigma-1)$ and $H^{\prime \prime}=H(\theta+\psi-1)$.

Suppose $\psi=\sigma$, then obviously

$$
\sum_{P \in S(H)} q^{|P|}=\left(\sum_{P^{\prime} \in S\left(H^{\prime}\right)} q^{\left|P^{\prime}\right|}\right) G\left(f_{\psi}-1, w_{\psi}\right) .
$$

By the inductive assumption, the right-hand side is equal to $\frac{1-q}{1-q^{\omega \eta-\theta+1}} \prod_{i>\eta-\theta} G\left(f_{i}, w_{i}-1\right)$. Suppose $\psi<\sigma$, then

$$
\begin{aligned}
& \sum_{P \in S(H)} q^{|P|}=\left(\sum_{P^{\prime} \in S\left(H^{\prime}\right)} q^{\left|P^{\prime}\right|}\right) G\left(f_{\sigma}, w_{\sigma}\right)+ \\
& \quad+\sum_{p=\sigma-k}^{\psi-1}\left(\sum_{\substack{P^{\prime \prime} \in S\left(H^{\prime \prime}\right) \\
\phi_{P^{\prime \prime}}(\psi)=p}} q^{\left|P^{\prime \prime}\right|}\right)\left(\prod_{i=\psi}^{\sigma-1} q^{f_{i}} G\left(f_{i}, w_{i}-1\right)\right) G\left(f_{\sigma}-1, w_{\sigma}\right) .
\end{aligned}
$$

By the inductive assumption, the right-hand side is equal to

$$
\begin{aligned}
& \frac{1-q}{1-q^{w_{\eta-\theta+1}}}\left[\prod_{i=\eta-\theta+1}^{\sigma-1} G\left(f_{i}, w_{i}-1\right)\right] \times \\
& \times\left(\frac{1-q^{\sum_{i=\psi}^{\sigma-1} f_{i}}}{1-q} G\left(f_{\sigma}, w_{\sigma}\right)+\frac{1-q^{\sum_{i=\sigma-k}^{\psi-1} f_{i}}}{1-q} q^{\sum_{i=\psi}^{\sigma-1} f_{i}} G\left(f_{\sigma}-1, w_{\sigma}\right)\right) .
\end{aligned}
$$

It is easy to check that it is equal to $\frac{1-q}{1-q^{w} \eta-\theta+1} \prod_{i>\eta-\theta} G\left(f_{i}, w_{i}-1\right)$. Hence, (5) is proved.

Let us prove (6). Suppose $p>\sigma$, then (6) is trivial because both sides are equal to zero. Suppose $p<\sigma$, then we have

$$
\sum_{\substack{P \in S(H) \\ \phi_{P}(\psi+k)=p}} q^{|P|}=\left(\sum_{\substack{P^{\prime} \in S\left(H^{\prime}\right) \\ \phi_{P^{\prime}}(\psi+k)=p}} q^{\left|P^{\prime}\right|}\right) q^{f_{\sigma}} G\left(f_{\sigma}, w_{\sigma}-1\right) .
$$

By the inductive assumption, the right-hand side is equal to $q^{\sum_{i=p+1}^{\psi+k-1} f_{i}} \frac{1-q^{f_{p}}}{1-q^{W} \psi+k}\left(\sum_{P \in S(H)} q^{|P|}\right)$. 
Suppose $p=\sigma$, then we have

$$
\begin{aligned}
& \sum_{\substack{P \in S(H) \\
\phi(\psi+k)=\sigma}} q^{|P|}=\left(\sum_{P^{\prime} \in S\left(H^{\prime}\right)} q^{\left|P^{\prime}\right|}\right) G\left(f_{\sigma}-1, w_{\sigma}\right)+ \\
& \quad+\sum_{u=\sigma-k}^{\psi-1}\left(\sum_{\substack{P^{\prime \prime} \in S\left(H^{\prime \prime}\right) \\
\phi_{P^{\prime \prime}}(\psi)=u}} q^{\left|P^{\prime \prime}\right|}\right)\left(\prod_{i=\psi}^{\sigma-1} q^{f_{i}} G\left(f_{i}, w_{i}-1\right)\right) G\left(f_{\sigma}-1, w_{\sigma}\right) .
\end{aligned}
$$

By the inductive assumption, the right-hand side is equal to

$$
\begin{aligned}
\frac{1-q}{1-q^{w_{\eta-\theta+1}}}\left[\prod_{i=\eta-\theta+1}^{\sigma-1} G\right. & \left.\left(f_{i}, w_{i}-1\right)\right] G\left(f_{\sigma}-1, w_{\sigma}\right) \times \\
& \times\left(\frac{1-q^{\sum_{i=\psi}^{\sigma-1} f_{i}}}{1-q}+\frac{1-q^{\sum_{i=\sigma-k}^{\psi-1} f_{i}}}{1-q} q^{\sum_{i=\psi}^{\sigma-1} f_{i}}\right) .
\end{aligned}
$$

It is easy to check that it is equal to $\frac{1-q^{f_{\sigma}}}{1-q^{w^{w} \psi+k}}\left(\sum_{P \in S(H)} q^{|P|}\right)$. Thus, (6) is proved. This completes the proof of the theorem.

\section{Proof of Theorem 1.5}

We need another description of the varieties $\left(\mathbb{C}^{2}\right)^{[N](k, n)}$. We define the discontinuous map $\rho:\left(\mathbb{C}^{2}\right)^{[N]} \rightarrow\left(\mathbb{C}^{2}\right)_{1, k}^{[N]}$ by the following formula $\rho(p)=\lim _{t \rightarrow 0} t p$, where $p \in\left(\mathbb{C}^{2}\right)^{[N]}$ and $t \in T_{1, k}$. It is easy to see that

$$
\left(\mathbb{C}^{2}\right)^{[N](k, n)}=\rho^{-1}\left(\coprod_{\substack{H=\left(d_{0}, d_{1}, \ldots\right) \\ \sum d_{i}=N, d_{\geq k n-k}=0}}\left(\mathbb{C}^{2}\right)_{1, k}^{[N]}(H)\right) .
$$

Clearly, the map $\rho^{-1}\left(\left(\mathbb{C}^{2}\right)_{1, k}^{[N]}(H)\right) \stackrel{\rho}{\rightarrow}\left(\mathbb{C}^{2}\right)_{1, k}^{[N]}(H)$ is a locally trivial bundle with an affine space as the fiber. We denote by $d_{1, k}^{+}(H)$ the dimension of the fiber. Therefore, we have

$$
\left[\left(\mathbb{C}^{2}\right)^{[N](k, n)}\right]=\sum_{\substack{H=\left(d_{0}, d_{1}, \ldots\right) \\ \sum d_{i}=N, d_{\geq k n-k}=0}}\left[\left(\mathbb{C}^{2}\right)_{1, k}^{[N]}(H)\right] \mathbb{L}^{d_{1, k}^{+}(H)}
$$

Consider the point $p \subset\left(\mathbb{C}^{2}\right)_{1, k}^{[N]}(H)$ corresponding to a monomial ideal I. From (1) it follows that

$$
\begin{array}{r}
d_{1, k}^{+}(H)=\left|\left\{s \in D_{I} \mid l(s)+1>k a(s)\right\}\right|+\left|\left\{s \in D_{I} \mid k(a(s)+1)>l(s)\right\}\right|= \\
=\left|D_{I}\right|+\left|\left\{s \in D_{I} \mid k a(s) \leq l(s)<k(a(s)+1)\right\}\right| .
\end{array}
$$


Obviously, the map $\pi \mapsto D_{\pi}$ is a bijection between the sets $L_{k n, n}^{+}$and $\left\{D \in \mathcal{D} \mid \operatorname{diag}_{\geq k n-k}^{1, k}(D)=0\right\}$. Hence, from (2) it follows that

$$
\begin{aligned}
& \sum_{N \geq 0}\left[\left(\mathbb{C}^{2}\right)^{[N](k, n)}\right] t^{N}=\sum_{\substack{D \in \mathcal{D} \\
\operatorname{diag}_{\geq k n-k}^{1, k}(D)=0}} \mathbb{L}^{|D|+|\{s \in D \mid k a(s) \leq l(s) \leq k(a(s)+1)\}|} t^{|D|}= \\
& =(\mathbb{L} t)^{\frac{k n(n-1)}{2}} \sum_{\pi \in L_{k n, n}^{+}} \mathbb{L}^{b_{k}(\pi)}(\mathbb{L} t)^{-\operatorname{area}(\pi)}=(\mathbb{L} t)^{\frac{k n(n-1)}{2}} C_{n}^{(k)}\left(\mathbb{L}, \mathbb{L}^{-1} t^{-1}\right) .
\end{aligned}
$$

This completes the proof of the theorem.

\section{Proof of Theorem 1.6}

We use the map $\rho:\left(\mathbb{C}^{2}\right)^{[N]} \rightarrow\left(\mathbb{C}^{2}\right)_{1, k}^{[N]}$ and the numbers $d_{1, k}^{+}(H)$ from the proof of Theorem 1.5. We have

$$
\left[\left(\mathbb{C}^{2}\right)^{[N]}\right]=\sum_{\substack{H=\left(d_{0}, d_{1}, \ldots\right) \\ \sum d_{i}=N}}\left[\left(\mathbb{C}^{2}\right)_{1, k}^{[N]}(H)\right] \mathbb{L}^{d_{1, k}^{+}(H)} .
$$

It is well known (see e.g.[18]) that

$$
\sum_{N \geq 0}\left[\left(\mathbb{C}^{2}\right)^{[N]}\right] t^{N}=\prod_{i \geq 1} \frac{1}{1-\mathbb{L}^{i+1} t^{i}}
$$

We know that a sequence $H$ is good if and only if $\left(\mathbb{C}^{2}\right)_{1, k}^{[N]}(H) \neq \emptyset$. The class $\left[\left(\mathbb{C}^{2}\right)_{1, k}^{[N]}(H)\right]$ is computed in Theorem 1.2 , so we only need to prove that if $\left(\mathbb{C}^{2}\right)_{1, k}^{[N]}(H) \neq \emptyset$, then

$$
d_{1, k}^{+}(H)=\sum_{i \geq 0} d_{i}+\sum_{i \geq \eta} e_{i}\left(\frac{k}{2}\left(e_{i}-1\right)+\sum_{j=1}^{k-1}(k-j) e_{i+j}\right),
$$

where $e_{i}=d_{i}-d_{i+1}+\tau(i)$. We prove (7) by induction on $N$. It is true for $N=0$. Suppose $N \geq 1$. Consider a point $p \subset\left(\left(\mathbb{C}^{2}\right)_{1, k}^{[N]}(H)\right)^{T}$. Let $D$ be the corresponding Young diagram. We have

$$
d_{1, k}^{+}(H)=|D|+|\{s \in D \mid k a(s) \leq l(s)<k(a(s)+1)\}| .
$$

There exists a unique point $p \in\left(\left(\mathbb{C}^{2}\right)_{1, k}^{[N]}(H)\right)^{T}$ such that the corresponding Young diagram $D$ satisfies the condition $\mid\{s \in D \mid l(s)=$ $k(a(s)+1)\} \mid=0$. It is equivalent to the fact that for any $i \geq 1$ we have $\left|\left\{j \in \mathbb{Z}_{\geq 0} \mid c_{j}(D)=i\right\}\right| \leq k$. Let $D^{\prime}=D(0,1)$ and $H^{\prime}=\operatorname{diag}^{1, k}\left(D^{\prime}\right)$. It is easy to see that

$$
\begin{aligned}
& |\{s=(i, j) \in D \mid j=0, k a(s) \leq l(s)<k(a(s)+1)\}|= \\
& \sum_{i=0}^{k-1}\left(d_{\eta-i}-d_{\eta-i+k}\right)=\sum_{i=0}^{k-1}(k-i) e_{\eta+i}-k .
\end{aligned}
$$


Therefore, we have

$$
\begin{aligned}
& |\{s \in D \mid k a(s) \leq l(s)<k(a(s)+1)\}|= \\
& \left|\left\{s \in D^{\prime} \mid k a(s) \leq l(s)<k(a(s)+1)\right\}\right|+\sum_{i=0}^{k-1}(k-i) e_{\eta+i}-k .
\end{aligned}
$$

By the inductive assumption, the right-hand side is equal to

$$
\begin{aligned}
& \sum_{i \geq \eta}\left(e_{i}-\delta_{i, \eta}\right)\left(\frac{k}{2}\left(e_{i}-\delta_{i, \eta}-1\right)+\sum_{j=1}^{k-1}(k-j) e_{i+j}\right)+\sum_{i=0}^{k-1}(k-i) e_{\eta+i}-k= \\
& \sum_{i \geq \eta} e_{i}\left(\frac{k}{2}\left(e_{i}-1\right)+\sum_{j=1}^{k-1}(k-j) e_{i+j}\right) .
\end{aligned}
$$

This completes the proof of the theorem.

\section{Homogeneous nested Hilbert schemes}

In this section we prove Theorem [1.7. In section 7.1 we recall the quiver descriptions of the varieties $\left(\mathbb{C}^{2}\right)_{1, k}^{[n]}(H)$ and $\left(\mathbb{C}^{2}\right)_{1,1}^{[\bar{n}]}(\bar{H})$. In section 7.2 we apply this description to conclude the proof of the theorem.

7.1. A quiver description. The variety $\left(\mathbb{C}^{2}\right)^{[n]}$ has the following description (see e.g.[18]).

$$
\left(\mathbb{C}^{2}\right)^{[n]} \cong\left\{\begin{array}{l|c}
1 & {\left[B_{1}, B_{2}\right]=0} \\
\left(B_{1}, B_{2}, i\right) & \begin{array}{c}
2)(\text { stability) There is no subspace } \\
S \subsetneq \mathbb{C}^{n} \text { such that } B_{\alpha}(S) \subset S(\alpha=1,2) \\
\text { and } \operatorname{im}(i) \subset S
\end{array}
\end{array}\right\} / G L_{n}(\mathbb{C}),
$$

where $B_{\alpha} \in \operatorname{End}\left(\mathbb{C}^{n}\right)$ and $i \in \operatorname{Hom}\left(\mathbb{C}, \mathbb{C}^{n}\right)$ with the action given by $g \cdot\left(B_{1}, B_{2}, i\right)=\left(g B_{1} g^{-1}, g B_{2} g^{-1}, g i\right)$, for $g \in G L_{n}(\mathbb{C})$.

Let $H=\left(d_{0}, d_{1}, \ldots\right)$. Let $V_{i}=\mathbb{C}^{d_{i}}$. It is easy to see that the variety $\left(\mathbb{C}^{2}\right)_{1, k}^{[n]}(H)$ has the following description (see Figure 4).

$$
\begin{aligned}
& \left(\mathbb{C}^{2}\right)_{1, k}^{[n]}(H) \cong \\
& \cong\left\{\begin{array}{l|l}
\left(\left(B_{1, j}, B_{2, j}\right)_{j \geq 0}, i\right) & \begin{array}{c}
1) B_{1, j+k} B_{2, j}-B_{2, j+1} B_{1, j}=0 \\
2) \text { There is no graded subspace } \\
S \subsetneq \bigoplus_{j \geq 0} V_{j} \text { such that } B_{\alpha}(S) \subset S \\
(\alpha=1,2) \text { and } i m(i) \subset S
\end{array}
\end{array}\right\} / \prod_{j \geq 0} G L_{d_{j}}(\mathbb{C}),
\end{aligned}
$$

where $B_{1, j} \in \operatorname{Hom}\left(V_{j}, V_{j+1}\right), B_{2, j} \in \operatorname{Hom}\left(V_{j}, V_{j+k}\right)$ and $i \in \operatorname{Hom}\left(\mathbb{C}, V_{0}\right)$.

Let $\bar{H}=\left(H_{1}, \ldots, H_{k}\right)$, where $H_{i}=\left(d_{i, 0}, d_{i, 1}, \ldots\right)$. Let $V_{i, j}=\mathbb{C}^{d_{i, j}}$. It is easy to see that the variety $\left(\mathbb{C}^{2}\right)_{1,1}^{[\bar{n}]}(\bar{H})$ has the following description 


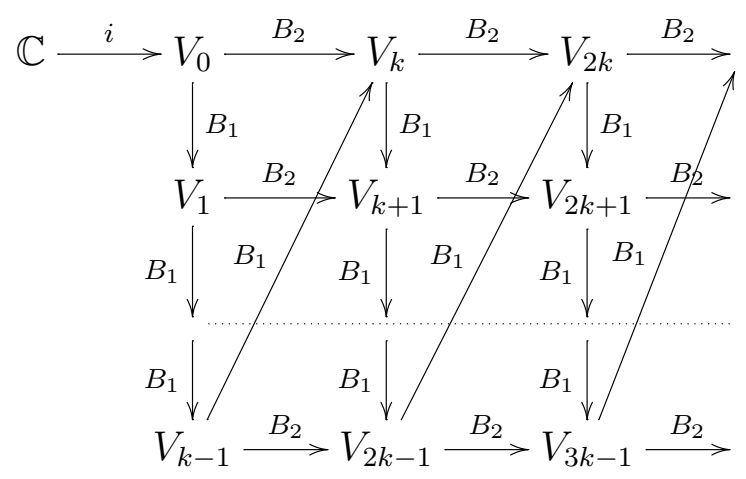

Figure 4. The quiver description of $\left(\mathbb{C}^{2}\right)_{1, k}^{[n]}(H)$

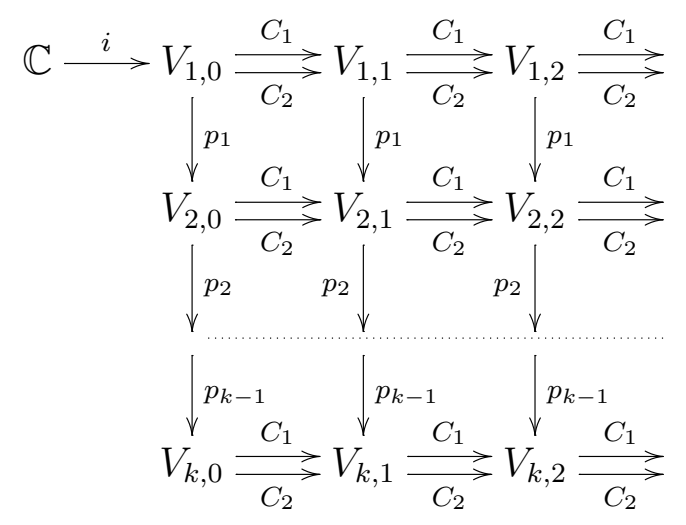

FiguRE 5. The quiver description of $\left(\mathbb{C}^{2}\right)_{1,1}^{[\bar{n}]}(\bar{H})$

(see Figure 5).

$$
\begin{aligned}
& \left(\mathbb{C}^{2}\right)_{1,1}^{[\bar{n}]}(\bar{H}) \cong \\
& \cong\left\{\left(\left(C_{1, j, h}, C_{2, j, h}\right)_{\substack{1 \leq j \leq k \\
0 \leq h}},\left(p_{j, h}\right)_{\substack{1 \leq j \leq k-1 \\
0 \leq h}}, i\right) \mid\right. \\
& \text { 1) } C_{1, j, h+1} C_{2, j, h}-C_{2, j, h+1} C_{1, j, h}=0 \\
& \left.\begin{array}{c}
\text { 2) } C_{\alpha, j+1, h} p_{j, h}-p_{j, h+1} C_{\alpha, j, h}=0 \\
\text { 3) There is no graded subspace } S \subsetneq \bigoplus_{j, h} V_{j, h} \\
\text { such that } B_{\alpha}(S) \subset S, p(S) \subset S \text { and } i m(i) \subset S
\end{array}\right\}=\prod_{j, h} G L_{d_{j, h}}(\mathbb{C}) \text {, }
\end{aligned}
$$

where $C_{\alpha, j, h} \in \operatorname{Hom}\left(V_{j, h}, V_{j, h+1}\right), p_{j, h} \in \operatorname{Hom}\left(V_{j, h}, V_{j+1, h}\right)$ and $i \in$ $\operatorname{Hom}\left(\mathbb{C}, V_{1,0}\right)$.

7.2. Proof of Theorem 1.7. We use the notations from section 1.8. Proposition 7.1. There is a natural map $\pi:\left(\mathbb{C}^{2}\right)_{1, k}^{[n]}(H) \rightarrow\left(\mathbb{C}^{2}\right)_{1,1}^{[\bar{n}]}(\bar{H})$.

Proof. Clearly, we have $V_{j, h}=V_{j-1+k h}$, for $1 \leq j \leq k, 0 \leq h$. We define the map $\pi$ by the following formula $\pi:\left(B_{1}, B_{2}, i\right) \mapsto\left(C_{1}, C_{2}, p, i\right)$, where $C_{1}=B_{1}^{k}, C_{2}=B_{2}, p=B_{1}$. 
Proposition 7.2. Under the conditions of Theorem 1.7, the map $\pi$ is an isomorphism.

Proof. From the stability condition and the commutation relations it follows that the map $p_{j, h}$ is an isomorphism if $d_{j, h}=d_{j+1, h}$. Let us define a map $\phi:\left(\mathbb{C}^{2}\right)_{1,1}^{[\bar{n}]}(\bar{H}) \rightarrow\left(\mathbb{C}^{2}\right)_{1, k}^{[n]}(H)$ by the following formula $\phi:\left(C_{1}, C_{2}, p, i\right) \mapsto\left(B_{1}, B_{2}, i\right)$, where $B_{2}=C_{2}$ and

$B_{1, j-1+k h}= \begin{cases}p_{j, h}, & \text { if } 1 \leq j \leq k-1, \\ C_{1,1, h} p_{1, h}^{-1} \ldots p_{k-2, h}^{-1} p_{k-1, h}^{-1}, & \text { if } j=k \text { and } h \in E(\bar{H}), \\ p_{1, h+1}^{-1} \ldots p_{k-2, h+1}^{-1} p_{k-1, h+1}^{-1} C_{1, j, h}, & \text { if } j=k \text { and } h+1 \in E(\bar{H}) .\end{cases}$

Clearly, the map $\phi$ is inverse to $\pi$.

Theorem 1.7 follows from these two propositions.

\section{REFERENCES}

[1] G. E. Andrews. The theory of partitions. Encyclopedia of Mathematics and its Applications, Vol. 2. Addison-Wesley Publishing Co., Reading, Mass.-LondonAmsterdam, 1976, 255 pp.

[2] A. Bialynicki-Birula. Some theorems on actions of algebraic groups. Ann. Math. 98, 480-497 (1973).

[3] A. Bialynicki-Birula. Some properties of the decompositions of algebraic varieties determined by actions of a torus. Bull. Acad. Pol. Sci. S6r. Sci. Math. astron. Phys. 24, (No. 9) 667-674 (1976).

[4] J. Cheah. Cellular decompositions for nested Hilbert schemes of points. Pacific J. Math. 183 (1998), no. 1, 39-90.

[5] K. Costello and I. Grojnowski. Hilbert schemes, Hecke algebras and the Calogero-Sutherland system. math.AG/0310189.

[6] G. Ellingsrud, S. A. Stromme. On the homology of the Hilbert scheme of points in the plane. Invent. math. 87, 343-352 (1987).

[7] L. Evain. Irreducible components of the equivariant punctual Hilbert schemes. Adv. Math. 185 (2004), no. 2, 328-346.

[8] S. M. Gusein-Zade, I. Luengo, A. Melle Hernandez. On generating series of classes of equivariant Hilbert schemes of fat points. Mosc. Math. J. 10 (2010), no. 3 .

[9] L. Gottsche. Hilbert schemes of points on surfaces. ICM Proceedings, Vol. II (Beijing, 2002), 483-494.

[10] J. Haglund. The $q$, $t$-Catalan Numbers and the Space of Diagonal Harmonics: With an Appendix on the Combinatorics of Macdonald Polynomials. University of Pennsylvania, Philadelphia - AMS, 2008, 167 pp.

[11] A. Iarrobino. Punctual Hilbert schemes. Mem. Am. Math. Soc., (188), 1977.

[12] A. Iarrobino, J. Yameogo. The family $G_{T}$ of graded artinian quotients of $k[x, y]$ of given Hilbert function. Special issue in honor of Steven L. Kleiman. Comm. Algebra 31 (2003), no. 8, 3863-3916.

[13] A. N. Kirillov. Combinatorics of Young tableaux and configurations. Proceedings of the St. Petersburg Mathematical Society, Vol. 7, 1798, Amer. Math. Soc. Transl. Ser. 2, 203, Amer. Math. Soc., Providence, RI, 2001.

[14] M. Lehn. Chern classes of tautological sheaves on Hilbert schemes of points on surfaces. Invent. Math. 136 (1999), no. 1, 157-207. 
[15] M. Lehn and C. Sorger. Symmetric groups and the cup product on the cohomology of Hilbert schemes. Duke Math. J. 110 (2001), no. 2, 345-357.

[16] W.-P. Li, Z. Qin, W. Wang, Vertex algebras and the cohomology ring structure of Hilbert schemes of points on surfaces. Math. Ann. 324 (2002), no. 1, 105-133.

[17] N. A. Loehr. Conjectured statistics for the higher $q, t$-Catalan sequences. Electron. J. Combin. 12 (2005), Research Paper 9, 54 pp.

[18] H. Nakajima. Lectures on Hilbert schemes of points on surfaces. AMS, Providence, RI, 1999.

[19] E. Vasserot, Sur lanneau de cohomologie du schema de Hilbert de $\mathbb{C}^{2}$, C. R. Acad. Sci. Paris Ser. I Math. 332 (2001), no. 1, 7-12.

Faculty of Mechanics and Mathematics, Moscow State University, 119991 Moscow, Russia AND

Department of Mathematics, University of Amsterdam, P. O. Box 94248, 1090 GE Amsterdam, The Netherlands

E-mail address: buryaksh@mail.ru, a.y.buryak@uva.nl 